\title{
Influencia del tratamiento penitenciario en la re-educación, rehabilitación, y reincorporación social de los reclusos del establecimiento penitenciario Pocollay de Tacna, periodo 2010- 2012
}

\author{
Influence of correctional treatment in the re-education, rehabilitation and social reintegration of \\ prison inmates Pocollay Tacna, period 2010-2012
}

Ritsy Marianella Supo García ${ }^{1}$

\begin{abstract}
RESUMEN
Objetivo: Determinar la influencia del Tratamiento Penitenciario en el cumplimiento de los derechos de re-educación, rehabilitación y reincorporación social de los reclusos del establecimiento penitenciario de Pocollay de Tacna, período 2010-2012. Para ello se estableció la siguiente hipótesis: En el establecimiento penitenciario de Pocollay de Tacna, el Tratamiento Penitenciario influye desfavorablemente en el cumplimiento de los derechos de reeducación, rehabilitación y reincorporación social de los reclusos, previstos en el artículo 139 inciso 22 de la Constitución Política del Perú.
\end{abstract}

Método: La forma de investigación es una Investigación Aplicada, porque está orientada a la aplicación de los conocimientos a la solución de un problema, pues confronta la teoría con la realidad. También es un tipo de Investigación Socio Jurídica porque se estudian los hechos y relaciones de orden social regulados por normas. Se adoptó el diseño no experimental (ex post facto) Descriptivo - Explicativo. Para tal propósito, se consideró la información obtenida a través de la aplicación del Cuestionario; y, la Cédula de Entrevista.

Resultados: Los datos obtenidos se tabularon y analizaron mediante tablas y gráficos. Una vez finalizada la fase de análisis e interpretación de los resultados se determinó que: En el establecimiento penitenciario de Pocollay de Tacna, el Tratamiento Penitenciario influye desfavorablemente.

Conclusión: En el establecimiento penitenciario de Pocollay de Tacna, el Tratamiento Penitenciario influye desfavorablemente en el cumplimiento de los derechos de re-educación, rehabilitación y reincorporación social de los reclusos, período 2010-2012.

\section{PALABRAS CLAVE}

Tratamiento penitenciario, re-educación, rehabilitación, reincorporación social.

\begin{abstract}
Objetive: Determine the influence Correctional Treatment in fulfilling the rights of reeducation, rehabilitation and social reintegration of the inmates of the prison of Pocollay of Tacna, 2010-2012.

Established the following hypothesis: in the prison of Pocollay of Tacna, prison Treatment compliance adversely affects the rights of reeducation, rehabilitation and social reintegration of prisoners, provided for in Article 139, paragraph 22 of the Constitution of Peru.

Method: The research is a form of applied research, because it is oriented in the application of knowledge to the solution of a problem, as the theory confronts reality. It is also a type of Socio Legal Research that examines the events and relationships of social order governed by rules, which adopted the nonexperimental design (ex post facto) Description Explanatory. For this purpose we considered the information obtained through the application of the questionnaire, and the Certificate of Interview.

Result: The data were tabulated and analyzed using tables and graphs. After the phase of analysis and interpretation of the results was determined that: In the prison of Pocollay of Tacna, Prison Treatment compliance adversely affects.

Conclusion: In the prison of Pocollay of Tacna, Prison Treatment compliance adversely affects the rights of re-education, rehabilitation and social reintegration of prisoners, period 2010 2012.
\end{abstract}

\section{KEYWORDS}

Prison treatment, rehabilitation, rehabilitation, social reintegration 


\section{Introducción}

El Tratamiento Penitenciario en el Perú tiene como objetivo, según Ramos, J. (2009), la re-educación, rehabilitación y reincorporación del interno a la sociedad. El tratamiento consiste en la utilización de métodos médicos, biológicos, psicológicos, psiquiátricos, pedagógicos -o más precisamente andragógicos- sociales, laborales, y aquellos que permitan obtener el objetivo del tratamiento de acuerdo a las características propias del interno.

Solís, A.(2008), afirma que el tratamiento penitenciario en las cárceles está aún lejos de cumplir con las exigencias mínimas de esta norma. Los factores son diversos, destacando: el insuficiente personal para realizar esta labor, la falta de recursos logísticos para desarrollar adecuadamente las tareas de tratamiento, las dificultades en la infraestructura, la clasificación de internos de acuerdo a determinadas variables (primarios, reincidentes, bandas organizadas, jóvenes, adultos, etc.), el trato del personal, entre otros.

Asimismo, Solís, A. (2008) argumenta que el tratamiento es "...progresivo y comprende el desarrollo de programas de resocialización del interno en forma individualizada y grupal según la naturaleza de la atención. Será aplicado en forma multidisciplinaria por los profesionales y técnicos de tratamiento, promoviendo la participación del interno, así como de instituciones públicas y privadas, la familia y la sociedad". Esta explicación, con más detalle, sobre el tratamiento, nos lleva a concluir que lamentablemente los planes y programas que se aplican no son los adecuados, pues la cárcel actual no rehabilita, sino genera mayor delincuencia y criminalidad.

\section{Materiales y métodos}

La población de estudio estará comprendida por los profesionales en derecho: (jueces, abogados, fiscales); y en cuanto al Establecimiento Penitenciario de Pocollay de Tacna, la población la comprenden el director y los reclusos.

\section{Tabla 1. POBLACIÓN Y MUESTRA}

\begin{tabular}{crr}
\hline $\begin{array}{c}\text { Profesionales en } \\
\text { materia judicial }\end{array}$ & Población & Muestra \\
\hline Jueces penales & 15 & 3 \\
\hline Fiscales penales & 48 & 10 \\
\hline Abogados & 1392 & 291 \\
\hline Reclusos & 653 & 242 \\
\hline Director & 1 & 1 \\
\hline Total & 2109 & 547 \\
\hline
\end{tabular}

Fuente: Administración del Módulo Penal de la Corte Superior de Justicia de Tacna, Oficina de Administración del Distrito Fiscal de Tacna, Colegio de Abogados de Tacna, Estadística proporcionada por el Establecimiento Penitenciario Pocollay de Tacna. Información al 31-12-2012.

Para la recolección de datos se recurrió a la aplicación del instrumento de medición del cuestionario, la cédula de entrevista.

El cuestionario aplicado a los profesionales en materia judicial (abogados, fiscales, jueces) se realizó de manera anónima durante los días 15 y 16 de abril. El cuestionario aplicado a los reclusos se realizó del 22 al 26 de abril del 2013.

Finalmente, la entrevista se aplicó al director del Establecimiento 
Penitenciario de Varones de Pocollay el 29 de abril del 2013.

\section{Resultados}

Resultados del cuestionario aplicado a los reclusos

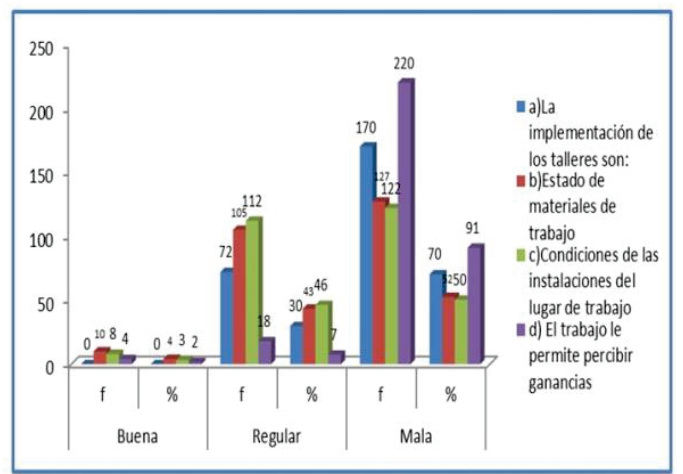

Figura 1.

Área de Trabajo

En la Figura 1 se observa que aproximadamente el $70 \%$ de los reclusos manifiesta que: la implementación de los talleres, el estado de materiales de trabajo, las condiciones de las instalaciones del lugar de trabajo, son "malas"; lo que no le permite percibir buenas ganancias, para satisfacer sus necesidades básicas y las de su familia.

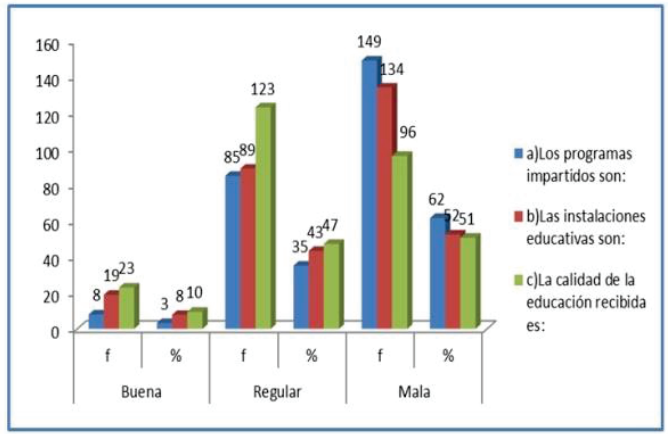

Figura 2.

Área de Educación

En la Figura 2 se observa que el $62 \%$ de los reclusos manifiesta que los programas impartidos, las instalaciones educativas; y la calidad de la educación recibida son "malas". Los reclusos manifiestan que es necesario ampliar los programas educativos con otras asignaturas, que les brinde el estímulo necesario para su re-socialización.

Resultados del cuestionario aplicado a los profesionales del derecho.

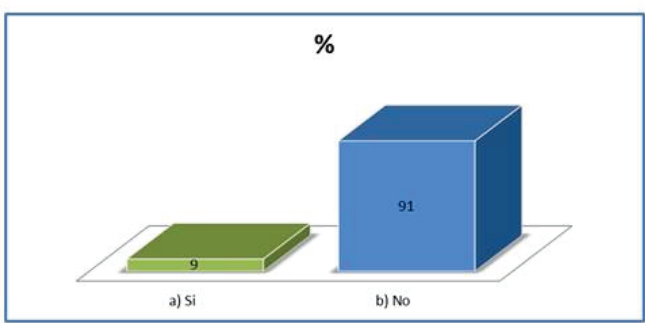

Figura 3.

¿Se cumple el principio que señala que el Tratamiento Penitenciario tiene por objeto la re-educación, rehabilitación y reincorporación social del penado a la sociedad?

En la Figura 3 se puede observar que el $91 \%$ de los encuestados señala que "no" se cumple el Principio de que el Tratamiento Penitenciario tenga por objeto la re-educación, rehabilitación y reincorporación social del penado a la sociedad; y, sólo el $09 \%$ señala lo contrario.

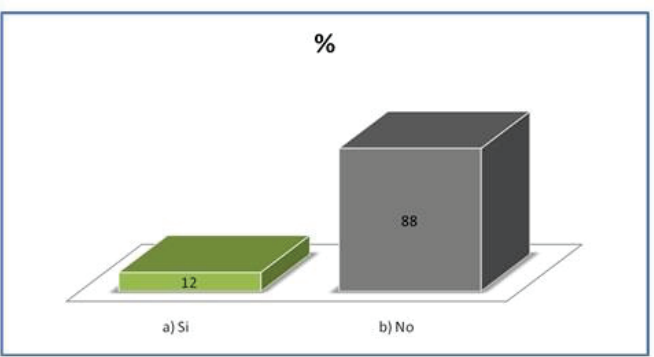

Figura 4.

¿Los programas educativos y laborales que actualmente imparte el INPE, son adecuados y/o suficientes para lograr la re-educación y rehabilitación de los internos? 
En la Figura 4 se observa que el $88 \%$ de los encuestados señala que los programas que actualmente imparte el INPE, no son adecuados y/o suficientes para lograr la re-educación de los internos; y sólo el $12 \%$ señala lo contrario.

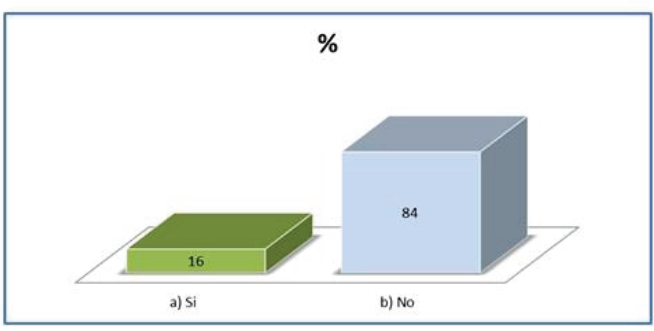

Figura 5.

¿La infraestructura actual con la que cuenta el Penal de Varones Pocollay garantiza la separación de reclusos de acuerdo a criterios de clasificación (primarios, con antecedentes, según edades)?

En la Figura 5 se puede observar que el $84 \%$ de los encuestados señala que la infraestructura actual con la que cuenta el Penal de Varones Pocollay "no" garantiza la separación de reclusos de acuerdo a criterios de clasificación (primarios, reos con antecedentes, por rangos de edad); y, sólo el $16 \%$ señala lo contrario.

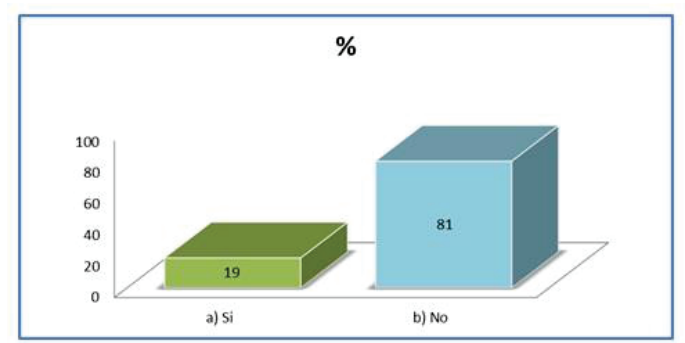

Figura 6.

¿Los internos que cumplen penas en el establecimiento penitenciario de varones de Pocollay logran su rehabilitación?
En la Figura 6 se observa que el $81 \%$ de los profesionales en derecho manifiesta que los internos que cumplen penas en el establecimiento penitenciario varones de Pocollay no ha logrado su rehabilitación; y, sólo el $19 \%$ señala lo contrario.

Análisis de resultados de documentos estadísticos

Tabla 2. POBLACIÓN PENAL POR ESPECIALIDAD EDUCATIVA EN EL ESTABLECIMIENTO PENITENCIARIO

\begin{tabular}{|l|c|}
\hline TIPO & TOTAL \\
\hline TOTAL & 212 \\
\hline A) INSTRUCCION EDUCATIVA & 62 \\
\hline ALFABETIZACION & 0 \\
\hline EDUCACION PRIMARIA & 22 \\
\hline EDUCACION SECUNDARIA & 40 \\
\hline B) CAPACITACION LABORAL & 150 \\
\hline ALBANILERIA & 0 \\
\hline ADMINISTRACION & 0 \\
\hline ARTESANIAS & 35 \\
\hline CARPINTERIAEN MADERA & 44 \\
\hline CARPINTERIAMETALICA & 0 \\
\hline CONTABILIDAD & 0 \\
\hline EBANISTERIA & 0 \\
\hline ELECTRICIDAD & 0 \\
\hline ELECTRONICA & 0 \\
\hline EDUCACIONFISICA, DEPORTEYRECREACION & 0 \\
\hline IDIOMAS & 0 \\
\hline INDUSTRIAALIMENTARIA & 0 \\
\hline INDUSTRIADEL VESTIDO (COnfecCIOnes) & 0 \\
\hline MECANICA AUTOMOTRIZ & 0 \\
\hline MECANICA DE BANCO & 0 \\
\hline PARAMEDICAS & 0 \\
\hline RADIO & 0 \\
\hline SASTRERIA & 28 \\
\hline SOLDADURAMECANICA & 0 \\
\hline TEJIDOSYUTE & 21 \\
\hline TORNO & 0 \\
\hline ZAPATERIA & \\
\hline OTROSYCOCINEROS & 0 \\
\hline
\end{tabular}

En el establecimiento penitenciario de Pocollay, al período 2012, de los 653 internos, por lo menos 62 se han inscrito en algún programa educativo; $y, 150$ de ellos se hallan inmersos en un programa de capacitación laboral.

En el establecimiento penitenciario de Pocollay no se dan las condiciones necesarias para contribuir a la resocialización, rehabilitación y 
reincorporación de los reclusos debido a que no se cuenta con programas suficientes para la alfabetización, Educación Básica Regular y ocupacional de los internos.

\section{Discusión}

El tratamiento es el elemento esencial del Sistema Penitenciario. El objetivo del tratamiento es la re-educación, rehabilitación y reincorporación del interno a la sociedad.

Todas estas disposiciones deben permitir se cumpla el objeto del Tratamiento Penitenciario el cual es la re-educación, rehabilitación y reincorporación social de los reclusos, derecho constitucional previsto en el artículo 139 inciso 22 de la Constitución Política del Perú. Sin embargo, en la actualidad, en el Establecimiento Penal de Varones Pocollay de la provincia de Tacna, se viene observando la existencia de condiciones inadecuadas de encarcelamiento e incumplimiento de normas de tratamiento penitenciario que repercuten en el fracaso de la resocialización de los internos de dicho penal.

La crisis del sistema penitenciario y las preocupaciones gubernamentales por encontrar alternativas de solución idóneas son una constante que aún espera respuesta adecuada. Dentro de este panorama crítico, tanto desde las vertientes valorativas y político sociales, así como desde los resultados de estudios empíricos, respecto al fin resocializador de la pena, nuestro Código de Ejecución Penal sigue la perspectiva de reeducar al interno a través de una política de tratamiento interdisciplinario. Sin embargo, entre la ley y la realidad fáctica del establecimiento penal de Pocollay aún hay un abismo que debe ser superado.

En la actualidad se tiene conocimiento que el penal tiene capacidad para 180 internos pero a la fecha alberga a 653; es decir, tiene una sobrepoblación de 473 internos. Por ello, la urgente necesidad de solucionar la problemática de este recinto que cuenta con 30 años de funcionamiento, y que requiere una adecuada infraestructura penitenciaria, así como la implementación de nuevos y novedosos programas, convenios y un marco legal adecuado acorde con nuestra realidad actual.

\section{Conclusiones}

- En el establecimiento penitenciario Pocollay de Tacna se da un inadecuado Tratamiento Penitenciario a los reclusos. Las autoridades han descuidado la asistencia al recluso en lo referido a las áreas de trabajo, educación, salud, asistencia psicológica y social.

- En el establecimiento penitenciario Pocollay de Tacna, no existen las condiciones necesarias, para lograr la re-educación, rehabilitación y reinserción de los reclusos, ya que no se cuenta con programas adecuados educativos y ocupacionales, no se prepara al interno para una actividad laboral que le provea adecuado sustento al egresar. La infraestructura no garantiza la separación de los reclusos por criterios de clasificación, entre otros factores. 
- En el establecimiento penitenciario Pocollay de Tacna, se incumple el principio del régimen penitenciario, y como parte de éste el tratamiento penitenciario que tiene por objeto la re-educación, rehabilitación y reincorporación del penado a la sociedad.

- En el establecimiento penitenciario de Pocollay de Tacna, el Tratamiento Penitenciario influye desfavorablemente en el cumplimiento de los derechos de reeducación, rehabilitación y reincorporación social de los reclusos.

- En la legislación peruana, no existen adecuadas normas que garanticen los derechos de re-educación, rehabilitación y reinserción de los reclusos, ya que existe ausentismo de normas prácticas que promuevan la participación del Gobierno Local y Regional a fin que realicen labores de apoyo al Tratamiento Penitenciario, y capacitación dentro de los penales. Asimismo, no se han dictado normas que fomenten la inversión privada, para convertir áreas de los penales en lugares de producción, manufacturas o insumos para el mercado.

\section{Referencias Bibliográficas}

1. Ramos, J. (2009). Derecho de Ejecución Penal y Ciencia Penitenciaria. Lima, Perú: Ediciones San Marcos EIRL.
2. Solís, A. (2008). Política Penal y Política Penitenciaria. Lima, Perú: Ediciones Departamento Académico de Derecho de la Pontificia Universidad Católica del Perú.

\section{E-mail: ritsysudro@hotmail.com}

Recibido: 04/04/14

Aceptado para publicación: 04/06/2014 